Pacific Journal of Mathematic 


\section{IDEMPOTENT MEASURES ON ABELIAN GROUPS}

WALTER RUDIN

\section{Introduction.}

1.1. All groups mentioned in this paper will be abelian, even when this is not explicitly stated, and will be written additively. For any locally compact abelian group $G$, IC $(G)$ will denote the set of all complex-valued Borel measures (sometimes called Radon measures) an $G$. The convolution of two such measures $\mu$ and $\lambda$ is the measure $\mu * \lambda$ defined by

$$
(\mu * \lambda)(E)=\int_{G} \mu(E-x) d \lambda(x)
$$

for every Borel set $E \subset G$, where $E-x$ is the set of all $y-x$ with $y \in E$.

With addition and scalar multiplication defined in the obvious way, and with convolution as multiplication, $\mathscr{C}(G)$ is a commutative algebra. A measure $\mu \in \mathscr{C l}(G)$ is said to be idempotent if

$$
\mu * \mu=\mu \text {. }
$$

The set of all idempotent elements of $\mathscr{C}(G)$ will be denoted by $\mathscr{I}(G)$.

It would be interesting to have an explicit description of the idempotent measures on any locally compact group. For the circle group, this was obtained by Helson [1], and was of considerable help in the determination of the endomorphisms of the group algebra of that group [6]. In the present paper, the problem is completely solved for the finitedimensional torus groups (Section IV) and for the discrete groups (Theorem 2.2). In Section II it is proved that every idempotent measure is concentrated on a compact subgroup (Theorem 2.1). In Section III the general problem is reduced to the study of the so-called irreducible idempotent measures on compact groups.

1.2. Apart from its intrinsic interest, this problem has another aspect: If $\mathscr{L}(G)$ is the Banach space of all complex Haar-integrable functions on $G$, and if $A$ is a bounded linear mapping of $\mathscr{L}(G)$ into $\mathscr{L}(G)$ which commutes with all translations of $G$, then it is known that there is a unique $\mu \in \mathscr{l}(G)$ such that

$$
(A f)(x)=\int_{G} f(x-y) d \mu(y)
$$

Furthermore, $A^{2}=A$ if and only if $\mu * \mu=\mu$.

Received May 22, 1958. The author is a Research Fellow of the Alfred P. Sloan Foundation. 
Thus the determination of all idempotents in $\mathscr{C}(G)$ is equivalent to the determination of all projections in $\mathscr{L}(G)$ which commute with the translations of $G$.

1.3. Let $\Gamma$ be the dual group of $G$, that is, the group of all continuous characters of $G$. With every $\mu \in \mathscr{C}(G)$ there is associated its Fourier-Stieltjes transform $\hat{\mu}$, defined by

$$
\hat{\mu}(\gamma)=\int_{G}(-x, \gamma) d \mu(x)
$$

where $(x, \gamma)$ denotes the value of the character $\gamma$ at the point $x$.

The correspondence $\mu \rightarrow \hat{\mu}$ is one-to-one, and the transform of a convolution is the pointwise product of the transforms of the factors. It follows that $\mu \in \mathscr{I}(G)$ if and only if $\hat{\mu}$ has 1 and 0 as its only values.

We associate with each $\mu \in \mathscr{I}(G)$ the set

$$
S(\mu)=\{\gamma \in \Gamma \mid \hat{\mu}(\gamma)=1\} .
$$

The problem of finding all $\mu \in \mathscr{F}(G)$ is then equivalent to the problem of finding all subsets of $\Gamma$ whose characteristic function is a FourierStieltjes transform.

1.4. In order to lead up to our conjecture concerning the structure of the measure in $\mathscr{J}(G)$, we present some relevant facts concerning: subgroups, quotient groups, and measures.

(y) If $H$ is a closed subgroup of $G$, let $N(H)$ be the annihilator of $H$, that is, the set of all $\gamma \in \Gamma$ such that $(x, \gamma)=1$ for all $x \in H$. Then $N(H)$ is a closed subgroup of $\Gamma$ and is the dual group of $G / H$. Also $\Gamma / N(H)$ is the dual group of $H$.

(b) For any $\mu \in \mathscr{C}(G)$, let $|\mu|$ be the measure defined by

$$
|\mu|(E)=\sup \sum\left|\mu\left(E_{i}\right)\right|,
$$

the sup being taken over all finite collections $\left\{E_{i}\right\}$ of pairwise disjoint Borel sets whose union is $E$; detailed proofs of the properties of the $|\mu|$ so defined can be found in [4]. The norm of $\mu$ is defined as $\|\mu\|=$ $|\mu|(G)$; with this norm, $\mathscr{C}(G)$ is a Banach algebra, and

$$
\|\mu\| \geq \sup |\hat{\mu}(\gamma)|
$$

$$
(\gamma \in \Gamma, \mu \in \mathscr{L}(G)) .
$$

We say that $\mu$ is concentrated on $E$ if $|\mu|(E)=\|\mu\|$. The restriction of $\mu$ to a set $A$ is the measure $\lambda$ defined by $\lambda(B)=\mu(A \cap B)$. The support of $\mu$ is the smallest closed set $F$ on which $\mu$ is concentrated. If $\mu$ is concentrated on a countable set, then $\mu$ is discrete; if $\mu(E)=0$ for every countable set $E, \mu$ is continuous; if $\mu(E)=0$ whenever $m(E)=0$, where $m$ is the Haar measure of $G, \mu$ is absolutely continuous; finally, if $\mu$ is concentrated on a set $E$ with $m(E)=0$, then $\mu$ is singular. 
(c) If a closed subgroup $H$ contains the support of some $\mu \in \mathscr{L}(G)$, then $\mu$ may also be regarded as an element of $\mathscr{l}(H)$. Conversely, any $\lambda \in \mathscr{l l}(H)$ may be regarded as an element of $\mathscr{C}(G)$, such that $\lambda(E)=0$, whenever $E \cap H$ is empty.

It is important to note that if a group $H$ contains the support of two measures $\mu, \lambda \in M(G)$, then the same is true of $\mu * \lambda$. This follows immediately from (1.1.1): Since $\lambda$ is concentrated on $H$, the integration extends over $H$ only, and if $E \cap H$ is empty, so is $(E-x) \cap H$ for every $x \in H$; hence $(\mu * \lambda)(E)=0$.

(d) If $H$ is a closed subgroup of $G$, if $\mu \in \mathscr{L l}(G)$, and if $\hat{\mu}$ is constant on each coset of $N(H)$, then $\mu$ is concentrated on $H$.

We sketch the proof. The assumption on $\mu$ implies that

$$
\int_{a}\left(-x, \gamma^{\prime}\right)(-x, \gamma) d \mu(x)=\int_{a}\left(-x, \gamma^{\prime}\right) d \mu(x) \quad\left(\gamma^{\prime} \in \Gamma, \gamma \in N(H),\right.
$$

and the uniqueness theorem for Fourier-Stieltjes transforms shows that $(-x, \gamma) d \mu(x)=d \mu(x)$ for all $\gamma \in N(H)$. Hence $(-x, \gamma)=1$ almost everywhere on the support of $\mu$, which means that this support lies in $H$.

(e) Suppose now that $G$ is compact (so that $\Gamma$ is discrete) and that $m_{H}$ is the Haar measure of a compact subgroup $H$ of $G$. Then $m_{I I} \in \mathscr{L C}(G)$ (see (c)), and it is easy to see that $m_{H} \in \mathscr{I}(G)$ and that $S\left(m_{H}\right)=N(H)$ (see (1.3.2)).

If, for some $\gamma \in \Gamma, d \mu(x)=(x, \gamma) d m_{H}(x)$, then $\mu$ is again idempotent, and $S(\mu)=N(H)+\gamma$.

It follows that every coset of every subgroup is $S(\mu)$ for some $\mu \in \mathscr{I}(G)$.

(f) Consider the family $W$ of all sets $S(\mu)$, for $\mu \in \mathscr{I}(G)$. If $\mu$ and $\lambda$ are idempotent, so are the measures $\mu * \lambda, \mu+\lambda-\mu * \lambda$, and $u-\mu$, where $u$ is the unit element of $\mathscr{C}(G)$ (i.e., $u$ is the point measure which assigns mass 1 to the identity elements of $G ; \hat{u}(\gamma)=1$ for all $\gamma \in \Gamma$ ). Since

$$
\begin{aligned}
& S(\mu) \cap S(\lambda)=S(\mu * \lambda), \\
& S(\mu) \cup S(\lambda)=S(\mu+\lambda-\mu * \lambda),
\end{aligned}
$$

and the complement of $S(\mu)$ is $S(u-\mu)$, we see that $W$ is closed under finite intersections, finite unions, and complementation. That is to say, $W$ is a ring of sets.

1.5. Suppose again that $G$ is compact. Define the coset-ring of $\Gamma$ to be the smallest ring of sets which contains all cosets of all subgroups of $\Gamma$. We conclude from 1.4 (e), (f), that every member of the coset-ring of $\Gamma$ is $S(\mu)$ for some $\mu \in \mathscr{F}(G)$.

The structure of such a $\mu$ can be described as follows: Every subgroup of $\Gamma$ is $N(H)$ for some compact subgroup $H$ of $G$, and any finite union of cosets of $N(H)$ is $S(\mu)$ for a measure $\mu$ defined by 


$$
d \mu(x)=\sum_{i=1}^{N}\left(x, \gamma_{i}\right) d m_{H}(x),
$$

where $\gamma_{1}, \cdots, \gamma_{N}$ are distinct characters of $H$. Roughly speaking, $\mu$ is a trigonometric polynomial on $H$. If $S(\lambda)$ belongs to the coset ring of $\Gamma, \lambda$ can accordingly be obtained from measures of the form (1.5.1) by a finite sequence of the operations described in 1.4 (f).

It seems quite likely that there are no other idempotent measures:

CONJECTURE. If $G$ is a compact abelian group and $\mu \in \mathscr{F}(G)$, then $S(\mu)$ belongs to the coset ring of $\Gamma$.

The main result of this paper is the proof of this conjecture for the finite-dimensional torus groups.

\section{Reduction to Compact Groups and Some Consequences ${ }^{1}$.}

Our first theorem shows that we may restrict our attention to measures defined on compact groups:

2.1. THeOREM. Suppose $G$ is a locally compact abelian group and $\mu \in \mathscr{I}(G)$. Then $\mu$ is concentrated on a compact subgroup $K$ of $G$ (and hence $\mu \in \mathscr{I}(K))$.

Proof. Let $G_{0}$ be the smallest closed subgroup of $G$ which contains the support of $\mu$; we wish to show that $G_{0}$ is compact. By 1.4(c) we may assume, without loss of generality, that $G_{0}=G$, and 1.4(d) implies then that $\hat{\mu}$ is not constant on the cosets of any non-trivial closed subgroup of $\Gamma$. In other words, if we define $\mu_{\gamma}$ by

$$
d \mu_{\gamma}(x)=(x, \gamma) d \mu(x)
$$

then $\mu_{\gamma} \neq \mu$ if $\gamma \neq 0$. Since $\hat{\mu}_{\gamma}=0$ or 1 , (1.4.2) implies

$$
\left\|\mu_{\gamma}-\mu\right\| \geq 1
$$$$
(\gamma \neq 0)
$$

There exists a compact set $C \subset G$ such that $|\mu|\left(C^{\prime}\right)<1 / 4$, where $C^{\prime}$ denotes the complement of $C$. If $V$ is the set of all $\gamma \in \Gamma$ such that

$$
|1-(x, \gamma)|<\frac{1}{3\|\mu\|}
$$

for every $x \in C$, then $V$ is open (this is precisely the way in which the topology of $\Gamma$ is defined), and for every $\gamma \in V$ we have

$$
\| \mu-\mu_{\gamma}|| \leq \int_{G}|1-(x, \gamma)| d|\mu|(x)=\int_{c}+\int_{c^{\prime}} \leq \frac{1}{3}+\frac{1}{2}<1 .
$$

1 The proofs in this section are simpler than they were in the original version of this paper, due to welcome suggestions by the referee and by P. J. Cohen. 
By (2.1.2), the open set $V$ thus consists of the identity element alone. Consequently, $I$ is discrete, and $G$ is compact.

2.2. Theorem. Suppose $G$ is discrete (so that $\Gamma$ is compact).

(a) If $\mu \in \mathscr{I}(G)$, then $\mu$ is concentrated on a finite subgroup $H$ of $G$, the annihilator $N(H)$ is an open-closed subgroup of $\Gamma$, and $S(\mu)$ is a finite union of cosets of $N(H)$.

(b) Conversely, every open-closed subset $E$ of $\Gamma$ is $S(\mu)$ for some $\mu \in \mathscr{J}(G)$.

Proof. The first part of (a) follows from Theorem 2.1; for the rest, we observe that $\Gamma / N(H)$ is a finite group and that $\hat{\mu}$ is constant on the cosets of $N(H)$.

Next, if $E$ is an open-closed subset of $\Gamma$, there is a neighborhood $V$ of 0 in $I$ such that $E+V=E$. If $f$ and $g$ are the characteristic functions of $V$ and $E$, respectively, then

$$
\int_{F} f\left(\gamma^{\prime}\right) g\left(\gamma-\gamma^{\prime}\right) d m\left(\gamma^{\prime}\right)=m(V) g(\gamma),
$$

where $m$ is the Haar measure of $\Gamma$. Since $f$ and $g$ are in $L^{2}(\Gamma)$, the Plancherel theorem implies that $g$ is a Fourier transform, and the result follows from the remark at the end of 1.3 .

2.3. For technical reasons, which will become apparent in the next section, it is convenient to enlarge the class $\mathscr{I}(G)$ somewhat. We let $\widetilde{F}(G)$ be the class of all $\mu \in \mathscr{l}(G)$ such that $\hat{\mu}$ is an integer-valued function, and we can immediately prove the following proposition:

If $\mu \in \mathscr{F}(G)$, then $\mu=a_{1} \mu_{1}+\cdots+a_{n} \mu_{n}$, where $a_{1}, \cdots, a_{n}$ are integers and $\mu_{1}, \cdots, \mu_{n} \in \mathscr{F}(G)$.

Indeed, let $a_{1}, \cdots, a_{n}$ be those integers which are different from 0 and which lie in the range of $\hat{\mu}$ (since $\hat{\mu}$ is bounded, this is a finite set). Let $P_{i}$ be a polynomial such that

$$
P_{i}(0)=0, P_{i}\left(a_{j}\right)=0 \text { if } j \neq i, P_{i}\left(a_{i}\right)=1,
$$

and put $\mu_{i}=P_{i}(\mu)$.

(We define $\mu^{n}=\mu * \mu^{n-1}$, and $P(\mu)=\sum_{1}^{k} c_{n} \mu^{n}$ if $P(x)=\sum_{1}^{k} c_{n} x^{n}$.) Then

$$
\hat{\mu}_{i}(\gamma)=P_{i}(\hat{\mu})(\gamma)= \begin{cases}1 & \text { if } \hat{\mu}(\gamma)=a_{i} \\ 0 & \text { otherwise }\end{cases}
$$

so that $\mu_{i} \in \mathscr{I}(G)$ and $\mu=a_{1} \mu_{1}+\cdots+a_{n} \mu_{n}$.

2.4. TheOREM. Suppose $G$ is a compact abelian group, $\mu \in \mathscr{F}(G)$, 
and $H$ is a closed subgroup of $G$. Let $\left\{H_{i}\right\}$ be the (evidently at most countable) collection of those cosets of $H$ for which

$$
|\mu|\left(H_{i}\right)>0 .
$$

Let $\tilde{H}$ be the smallest subgroup of $G$ which contains all these $H_{i}$, and let $\sigma$ be the restriction of $\mu$ to $\tilde{H}$. Then

(i) $\sigma \in \mathscr{F}(G)$;

(ii) $\tilde{H} / H$ is a finite group.

Assertion (ii) implies, in particular, that $\left\{H_{i}\right\}$ is a finite collection, and that $\mu$ vanishes on every coset of $H$ which has infinite order in $G / H$.

Proof. We first claim that the following two statements are true for every Borel set $A \subset G$ :

(a) If $A \cap \tilde{H}$ is empty, then $\sigma^{n}(A)=0$ for $n=1,2,3, \cdots$.

(b) If $A \subset \tilde{H}$, then $\sigma^{n}(A)=\mu^{n}(A)$ for $n=1,2,3, \cdots$.

Note that $\tilde{H}$ is an at most countable union of cosets of $H$, hence in particular is a Borel set.

It is clear that (a) holds if $n=1$, and we proceed by induction:

$$
\sigma^{n}(A)=\int_{a} \sigma^{n-1}(A-x) d \sigma(x)=\int_{\tilde{H}} \sigma^{n-1}(A-x) d \sigma(x) .
$$

If $A \cap \tilde{H}=0$ and $x \in \tilde{H}$, then $(A-x) \cap \tilde{H}=0$. Thus if (a) holds for $n-1$, it also holds for $n$.

To prove (b), put $\tau=\mu-\sigma$, and expand $\mu^{n}=(\sigma+\tau)^{n}$ by the binomial theorem. We have to show that

$$
\left(\tau^{k} * \sigma^{n-k}\right)(A)=0
$$$$
(k=1,2, \cdots, n)
$$

if $A \subset \tilde{H}$.

Since $\tau$ vanishes on every coset of $H$ and since $\tilde{H}$ is an at most countable union of such cosets, we have $\tau(A-x)=0$ for every $x \in G$. Thus, for any $\lambda \in \mathscr{M}(G)$,

$$
(\tau * \lambda)(A)=\int_{a} \tau(A-x) d \lambda(x)=0,
$$

and (2.4.2) follows.

From (a) and (b) we conclude that

$$
\sigma^{n}(E)=\mu^{n}(E \cap \tilde{H}) \quad(n=1,2,3, \cdots)
$$

for every Borel set $E \subset G$.

Let $a_{1}, \cdots, a_{n}$ be the non-zero values of $\hat{\mu}$, and let $P$ be the polynomial 


$$
P(t)=t \prod_{i=1}^{n}\left(t-a_{i}\right) .
$$

Then $P(\mu)=0$, and (2.4.3) implies that $P(\sigma)=0$. From this it follows that every value of $\hat{\sigma}$ is a root of $P$, so that the range of $\hat{\sigma}$ lies in the set $\left\{0, a_{1}, \cdots, a_{n}\right\}$. This proves the first part of the theorem.

We now put a new topology on $\tilde{H}$ : the neighborhoods of 0 are to be the sets $H \cap V$, for any neighborhood $V$ of 0 in $G$. Then $\tilde{H}$ is a locally compact abelian group, and $\sigma \in \mathscr{F}(\tilde{H})$. By Theorem 2.1 (extended from $\mathscr{F}(G)$ to $\mathscr{F}(G)$, via Proposition 2.3), we see that $\sigma$ is concentrated on a compact subgroup $K$ of $\tilde{H}$. The minimality which was one of the defining properties of $\tilde{H}$ shows that $K=\tilde{H}$. Thus $\tilde{H}$ is compact, and since $H$ is an open subgroup of $\tilde{H}$, we conclude that $\tilde{H} / H$ is finite.

\section{Decomposition into Irreducible Measures.}

3.1. If $G$ is compact, $\mu \in \mathscr{I}(G)$, and $H$ is a compact subgroup of $G$, the second part of Theorem 2.4 shows that $H$ has finite index in a compact subgroup $\tilde{H}$ such that $\mu$ vanishes on every translate of $\tilde{H}$ which is different from $H$. The existence of such $\tilde{H}$ suggests the following definitions.

$(\alpha)$ Suppose $G$ is compact, $\mu \in \mathscr{L}(G)$, and $K$ is a compact subgroup of $G$. We say that $K$ is associated with $\mu$ if

(i) $|\mu|(K+x)=0$ for every $x \notin K$;

(ii) $|\mu|(H)<|\mu|(K)$ for every compact subgroup $H$ of $K$ which is different from $K$.

Note that (ii) implies $|\mu|(K)>0$. Thus the null-measure has no group associated with it. If $\mu \neq 0$, the smallest compact group on which $\mu$ is concentrated is clearly associated with $\mu$.

( $\beta$ ) If $\mu=0$, or if there is precisely one compact subgroup associated with $\mu$, we say that $\mu$ is irreducible.

It should be pointed out that $\mu$ need not be concentrated on a subgroup which is associated with $\mu$. For example, let $G$ be the circle group $T^{1}$ (the one-dimensional torus), and set $\mu=m+\lambda$, where $m$ is the Haar measure of $T^{1}$, and $\lambda$ is a positive measure concentrated at the point $e^{i x}$. If $x / \pi$ is rational, then the finite cyclic group generated by $e^{i x}$ is associated with $\mu$, and so is $T^{1}$. If $x / \pi$ is irrational, then $\mathrm{T}^{1}$ is the only group associated with $\mu$, and $\mu$ is irreducible.

3.2. The following two propositions may elucidate these concepts (we use the notations of 3.1):

(a) If $K$ and $H$ are associated with $\mu$ and if $H$ is a proper subgroup of $K$, then $K / H$ is infinite. 
Indeed, suppose $K / H$ is finite, so that a finite number of translates of $H$ covers $K$. Since $|\mu|(H+x)=0$ whenever $x \notin H$, it follows that $|\mu|(K)=|\mu|(H)$, a contradiction.

(b) Suppose $\mu \in \mathscr{F}(G)$ and $K$ is the smallest compact subgroup of $G$ such that $|\mu|(K)=\|\mu\|$. Then $\mu$ is irreducible if and only if $|\mu|(H)=0$ for every compact subgroup $H$ of $K$ such that $m(H)=0$, where $m$ is the Haar measure of $K$. If $\mu$ is irreducible, we also have $|\mu|(H+x)=0$ for every such $H$ and all $x \in G$.

Note that $m(H)=0$ if and only if $K / H$ is infinite. Thus if $|\mu|(H)=0$ whenever $m(H)=0$, proposition (a) implies that $\mu$ is irreducible. On the other hand, if $|\mu|(H)>0$ for some $H$ with $m(H)=0$, the $\tilde{H}$ of Theorem 2.4 (with $K$ in place of $G$ ) is associated with $\mu$ and $m(\tilde{H})=0$; hence $\mu$ is not irreducible. The last assertion of (b) also follows from Theorem 2.4 .

3.3 THEOREM. Suppose $G$ is a compact abelian group and $\mu \in \mathscr{I}(G)$. Then there exist irreducible measures $\mu_{i} \in \mathscr{I}(G)$ and integers $a_{i}$ such that

$$
\mu=a_{1} \mu_{1}+a_{2} \mu_{2}+\cdots+a_{n} \mu_{n} .
$$

Proof. It is convenient to weaken the hypothesis somewhat and to assume merely that $\mu \in \mathscr{F}(G)$; assume also $\mu \neq 0$.

We first show that there is a compact subgroup $H_{1}$ of $G$ which is associated with $\mu$, such that the restriction $\lambda_{1}$ of $\mu$ to $H_{1}$ is irreducible, and such that $\lambda_{1} \in \mathscr{F}(G)$.

If $\mu$ is irreducible, let $H_{1}$ be the smallest compact group on which $\mu$ is concentrated. If $\mu$ is not irreducible, there is a compact group $K_{1}$ which is associated with $\mu$, such that $|\mu|\left(K_{1}\right)<\|\mu\|$; let $\sigma_{1}$ be the restriction of $\mu$ to $K_{1}$. By Theorem 2.4, $\sigma_{1} \in \mathscr{F}(G)$, and the same is thus true of $\mu-\sigma_{1}$. Since $\mu-\sigma_{1} \neq 0$, (1.4.2) shows that

$$
\left\|\mu-\sigma_{1}\right\| \geq 1 \text {. }
$$

On the other hand, $\sigma_{1}$ and $\mu-\sigma_{1}$ are concentrated on disjoint sets, so that

$$
\|\mu\|=\left\|\sigma_{1}\right\|+\left\|\mu-\sigma_{1}\right\|
$$

It follows that

$$
\left\|\sigma_{1}\right\| \leq\|\mu\|-1
$$

If $\sigma_{1}$ is not irreducible, we repeat this process, with $\sigma_{1}$ in place of $\mu$ : there is a compact $K_{2} \subset K_{1}$ which is associated with $\sigma_{1}$ (hence with $\mu$ ), such that the restriction $\sigma_{2}$ of $\sigma_{1}$ to $K_{2}$ belongs to $\mathscr{F}(G)$ and satisfies the inequality 


$$
\left\|\sigma_{2}\right\| \leq\left\|\sigma_{1}\right\|-1 \leq\|\mu\|-2 .
$$

Since the norms decrease by at least 1 each time, we evidently obtain an irreducible measure $(\neq 0)$ after repeating this process a finite number of times. Call this measure $\lambda_{1}$.

Since $\lambda_{1}$ is the restriction of $\mu$ to a group $H_{1}$, we see that $\lambda_{1}$ and $\mu-\lambda_{1}$ are concentrated on disjoint sets, so that $\|\mu\|=\left\|\lambda_{1}\right\|+\left\|\mu-\lambda_{1}\right\|$. We conclude, as above, that

$$
\left\|\mu-\lambda_{1}\right\| \leqq\|\mu\|-1 \text {. }
$$

If $\mu-\lambda_{1}$ is irreducible, put $\lambda_{2}=\mu-\lambda_{1}$. If not, repeat the preceding construction, finding an irreducible $\lambda_{2} \in \mathscr{F}(G)$ such that

$$
\left\|\mu-\lambda_{1}-\lambda_{2}\right\| \leq\|\mu\|-2 \text {. }
$$

Again, this must stop after a finite number of steps, and we obtain a representation

$$
\mu=\lambda_{1}+\lambda_{2}+\cdots+\lambda_{p},
$$

where each $\lambda_{i}$ is irreducible and belongs to $\mathscr{F}(G)$.

In 2.3, we saw that every $\lambda \in \mathscr{F}(G)$ is a finite linear combination, with integer coefficients, of idempotent measures $\mu_{j}$. The theorem thus follows from (3.3.7) if we can show that the $\mu_{j}$ are irreducible if $\lambda$ is irreducible; by 2.3 , we therefore have to show that $P(\lambda)$ is irreducible if $P$ is a polynomial without constant term.

Suppose then that $K$ is associated with $\lambda$ and that $\lambda$ is irreducible. Let $m$ denote the Haar measure of $K$. If $H$ is a compact subgroup of $K$ with $m(H)=0$, then $|\lambda|(H)=0$ by $3.2(\mathrm{~b})$, and it easily follows that $\left|\lambda^{n}\right|(H)=0$ for $n=1,2,3, \cdots$, and hence that $|P(\lambda)|(H)=0$. Thus if $P(\lambda) \neq 0, P(\lambda)$ is concentrated on a subgroup $K^{\prime}$ of $K$ which has finite index in $K$, and $K^{\prime}$ is associated with $P(\lambda)$. Applying 3.2 (b) again, we conclude that $P(\lambda)$ is irreducible.

This completes the proof.

3.4 Let $R$ denote the coset ring of $\Gamma$, and call a function $f$ defined on $\Gamma$ an $R$-function if

$$
f(\gamma)=\sum_{i=1}^{N} c_{i} g_{i}(\gamma)
$$

where each $g_{i}$ is the characteristic function of some member of $R$, and each $c_{i}$ is a complex number.

Suppose we know, for some compact group $G$, that $S(\mu) \in R$ for every irreducible $\mu \in \mathscr{I}(G)$. Then $\hat{\mu}$ is an $R$-function, and since a finite sum of $R$-functions is again an $R$-function, Theorem 3.3 shows that $\hat{\sigma}$ is an $R$-function for every $\sigma \in \mathscr{J}(G)$ (irreducible or not); this means 
that $S(\sigma) \in R$.

Thus the conjecture made in 1.5 will be proved for a given compact $G$ if it is proved for every irreducible $\mu \in \mathscr{I}(G)$.

According to the remarks made in 1.5, we therefore have to prove the following: if $K$ is the subgroup associated with the irreducible $\mu \in \mathscr{I}(G)$, then $\mu$ is a trigonometric polynomial on $K$. This is equivalent to the assertion that $\mu$ is absolutely continuous with respect to the Haar measure of $K$; for if $\mu$ is not a trigonometric polynomial, then $\hat{\mu}(\phi)=1$ for infinitely many $\phi$ in the dual group of $K$, so that $\hat{\mu}(\phi)$ does not vanish at infinity.

\section{The Idempotent Measure of the Torus Groups.}

4.1. Let $T^{r}$ denote the $r$-dimensional torus group; the points of $T^{r}$ are of the form

$$
x=\left(\xi_{1}, \cdots, \xi_{r}\right),
$$

the $\xi_{i}$ being real numbers mod $2 \pi$. The dual group of $T^{r}$ is $A^{r}$, the group of all lattice points in $r$-dimensional euclidean space $R^{r}$, i.e., the set of all

$$
n=\left(\nu_{1}, \cdots, \nu_{r}\right)
$$

where the $\nu_{i}$ are integers. If we put

$$
n \cdot x=\sum_{i=1}^{r} \nu_{i} \xi_{i},
$$

then $(x, n)=e^{i n \cdot x}$, and the transform of any $\mu \in \mathscr{C l}\left(T^{r}\right)$ is

$$
\hat{\mu}(n)=\int_{r^{r}} e^{-i n \cdot x} d \mu(x)
$$

We shall prove that every $\mu \in \mathscr{I}\left(T^{r}\right)$ has the structure described in 1.5 :

4.2. THEOREM. If $\mu \in \mathscr{J}\left(T^{r}\right)$, then $S(\mu)$ belongs to the coset ring of $\Lambda^{r}$.

The discussion in 3.4 shows that Theorem 4.2 is a consequence of the following:

4.3 THEOREM. Suppose $G$ is a compact subgroup of $T^{r}$, and suppose $G$ is associated with an irreducible measure $\mu \in \mathscr{J}\left(T^{r}\right)$. Then $\mu$ is absolutely continuous with respect to the Haar measure of $G$.

Proof. We shall use induction on $r$. 
For $r=1$, the theorem is due to Helson [1] (our terminology differs from his, being adapted to a more general situation), but we include the proof for the sake of completeness.

If $G$ is a finite group, there is nothing to prove. If $G=T^{1}$, we have to prove that $S(\mu)$ is finite. Since $\mu$ is continuous, a well-known theorem of Wiener implies that

$$
\lim _{N \rightarrow \infty} \frac{1}{2 N+1} \sum_{-N}^{N}|\hat{\mu}(n)|^{2}=0 .
$$

If $S(\mu)$ were infinite, it would therefore contain an infinite set $\left\{n_{k}\right\}$ such that none of the integers $n_{k}+1, \cdots, n_{k}+k$ belong to $S(\mu)$, and a subsequence of the measures $\mu_{k}$ defined by

$$
d \mu_{k}(x)=e^{-i n} k^{x} d \mu(x)
$$

would converge weakly (as functionals on the space of all continuous functions on $T^{1}$ ) to a measure $\sigma \in \mathscr{F}\left(T^{1}\right)$. The choice of $\left\{n_{k}\right\}$ shows that $\hat{\sigma}(0)=1$ and $\hat{\sigma}(n)=0$ for all $n>0$. This latter fact implies, by a well-known theorem of F. and M. Riesz, that $\sigma$ is absolutely continuous. But every weak limit of the sequence (4.3.2) must be singular [2; p. 236]. Since $\sigma \neq 0$, this is a contradiction, and we conclude that $S(\mu)$ is finite.

We now assume that the theorem has been proved for $r \leq p-1$ $(p=2,3,4, \cdots)$. To prove it for $r=p$, we consider two possibilities: F.

Case 1. G is the direct sum of $T^{q}($ for some $q<p)$ and a finite group

Case 2. $G=T^{p}$.

Case 1. Let $f_{1}, \cdots, f_{s}$ be the elements of $F$, so that each element of $G$ can be written uniquely in the form $x+f$ with $x \in T^{a}$ and $f \in F$. Let $\phi_{1}, \cdots, \phi_{s}$ be the characters of $F$.

Let $\mu_{1}, \cdots, \mu_{s}$ be measures on $T^{q}$ defined by

$$
\mu_{k}(E)=\sum_{j=1}^{s}\left(-f_{j}, \phi_{k}\right) \mu\left(E_{j}\right) \quad(k=1, \cdots, s),
$$

where $E$ is a Borel set in $T^{q}$ and $E_{j}=E+f_{j}$. Then, for $n \in \Lambda^{q}$, we have

$$
\begin{aligned}
\hat{\mu}_{k}(n) & =\int_{T^{q}} e^{-i n \cdot x} d \mu_{k}(x) \\
& =\sum_{j=1}^{s} \int_{T^{q_{+}}} e^{-i n \cdot x}\left(-f_{j}, \phi_{k}\right) d \mu\left(x+f_{j}\right) \\
& =\int_{G} e^{-i n \cdot x}\left(-f, \phi_{k}\right) d \mu(x+f)=\hat{\mu}\left(n+\phi_{k}\right)=0 \text { or } 1,
\end{aligned}
$$


so that $\mu_{k} \in \mathscr{J}\left(T^{q}\right)$.

Since $\mu$ is irreducible and $G$ is associated with $\mu$, (4.3.3) shows that $\left|\mu_{k}\right|(H)=0$ for every proper compact subgroup of $T^{q}$; hence $\mu_{k}$ is irreducible and $T^{\alpha}$ is associated with $\mu_{k}$, or $\mu_{k}=0$. Our induction hypothesis now implies that $\mu_{k}$ is absolutely continuous $(1 \leq k \leq s)$.

If we multiply the equations (4.3.3) by $\left(f_{i}, \phi_{k}\right)$, add over $k$, and observe the orthogonality relations satisfied by the characters $\phi_{k}$, we obtain

$$
\sum_{k=1}^{s}\left(f_{i}, \phi_{k}\right) \mu_{k}(E)=s \cdot \mu\left(E_{i}\right) \quad(i=1, \cdots, s),
$$

and thus the absolute continuity of the measures $\mu_{k}$ implies that $\mu$ is absolutely continuous with respect to the Harr measure of $G$. This settles Case 1.

Case 2. We now assume that $\mu \in \mathscr{F}\left(T^{p}\right)$ and that $|\mu|(H)=0$ on every proper compact subgroup of $T^{p}$ (compare 3.2 (b)), and we wish to prove that $S(\mu)$ is finite. Our proof will be similar to that of the case $r=1$, but we have to replace our reference to the theorem of $F$. and M. Riesz by a result recently proved by Helson and Lowdenslager ${ }^{1}$ [3; Section 4, Lemma 3]:

Let $Q$ be a subset of $\Lambda^{p}$ such that (a) $n_{1}+n_{2} \in Q$ if $n_{1} \in Q$ and $n_{2} \in Q$, (b) $0 \notin Q$, (c) if $n \neq 0$, then $n \in Q$ if and only if $-n \notin Q$. Suppose $\sigma$ is a singular measure on $T^{p}$ such that $\hat{\sigma}(n)=0$ for all $n \in Q$. Then $\hat{\sigma}(0)=0$.

We think of $\Lambda^{p}$ as a subset of euclidean space $R^{p}$ (see 4.1). The theorem of Wiener (namely, (4.3.1)) extends without difficulty to FourierStieltjes series in several variables and shows that $S(\mu)$ has density 0 in $\Lambda^{p}$, since $\mu$ is continuous. More precisely, the number of points of $S(\mu)$ in the $p$-dimensional cube with center at the origin and vertices $( \pm N, \pm N, \cdots \pm N)$ is $o\left(N^{p}\right)$.

If $S(\mu)$ is infinite, it follows that there exist spheres $V_{k}$ in $R^{p}$ with the following properties:

(i) The radius of $V_{k}$ is greater than $k$.

(ii) $V_{k}$ contains no point of $S(\mu)$ in its interior.

(iii) The boundary of $V_{k}$ contains a point $n_{k} \in S(\mu)$, and $n_{k} \rightarrow \infty$ as $k \rightarrow \infty$. (iv) If $c_{k}$ is the center of $V_{k}$, the unit vectors

$$
\frac{c_{k}-n_{k}}{\left|c_{k}-n_{k}\right|}
$$

converge to some $b \in R^{p}$. (The absolute value sign denotes the length of the vector.)

${ }^{1}$ I wish to thank these two authors for letting me read their paper prior to its publication. 
(v) The measures $\mu_{k} \in \mathscr{J}\left(T^{p}\right)$ defined by

$$
d \mu_{k}(x)=e^{-i n_{k} \cdot x} d \mu(x)
$$

converge weakly to a measure $\sigma \in \mathscr{J}\left(T^{p}\right)$.

It is evident that $\left\{V_{k}\right\}$ can be chosen so as to statisfy (i), (ii), (iii), and a suitable subsequence will satisfy (iv) and (v) as well.

The passage from $\mu$ to $\sigma$, via (4.3.5), is such that $|\sigma|(E) \leq|\mu|(E)$ for every Borel set $E \subset T^{p}$; also, $\sigma$ is not changed if we replace $\mu$ by its singular component, since the Fourier transform of the absolutely continuous component tends to 0 (this is the argument used by Helson in $[2 ; \mathrm{p} .236])$.

Hence $\sigma$ is singular, $\sigma \in \mathscr{F}\left(T^{p}\right)$, and since $|\sigma|(H) \leq|\mu|(H)=0$ for every proper compact subgroup $H$ of $T^{p}, \quad \sigma$ is irreducible; our choice of $\left\{V_{k}\right\}$ shows that $\hat{\sigma}(0)=1$ and that $\hat{\sigma}(n)=0$ for every $n$ in the open half-space determined by $n \cdot b>0$.

This last property is a consequence of the relation

$$
S\left(\mu_{k}\right)=S(\mu)-n_{k}
$$

and the fact that $S\left(\mu_{k}\right)$ therefore has no point in the interior of the sphere through 0 whose center is at $c_{k}-n_{k}$.

Let $\Lambda^{q}$ be the subgroup of $\Lambda^{p}$ which lies in the hyperplane $n \cdot b=0$. Then $0 \leq q<p$. If $q=0$, the theorem of Helson and Lowdenslager gives an immediate contradiction: take for $Q$ the set of all $n$ such that $n \cdot b>0$; then $\hat{\sigma}(n)=0$ in $Q, \sigma$ is singular, but $\hat{\sigma}(0)=1$.

If $0<q<p$, then $T^{p}$ is a direct sum $T^{q}+T^{p-q}$, where $\Lambda^{q}$ is the dual group of $T^{q}$ and the annihilator of $T^{p-q}$. Let $h$ be the natural homomorphism of $T^{p}$ onto $T^{q}$, and define a measure $\lambda \in \mathscr{C l}\left(T^{q}\right)$ by

$$
\lambda(E)=\sigma\left(h^{-1}(E)\right)
$$

for all Borel sets $E \subset T^{q}$. Any $x \in T^{p}$ has a unique representation $x=x_{1}+x_{2}$ with $x_{1} \in T^{q}, x_{2} \in T^{p-q}$. If $n \in \Lambda^{q}$, then $e^{i n \cdot x_{2}}=1$, so that

$$
\hat{\lambda}(n)=\int_{T^{q}} e^{-i n \cdot x_{2}} d \lambda\left(x_{1}\right)=\int_{T^{p}} e^{-i n \cdot x} d \sigma(x)=\hat{\sigma}(n)=0 \text { or } 1 .
$$

Thus $\lambda \in \mathscr{F}\left(T^{q}\right)$ and the irreducibility of $\sigma$ shows that $\lambda$ vanishes on every proper compact subgroup of $T^{q}$. Our induction hypothesis now implies that $S(\lambda)$ is a finite subset of $\Lambda^{q}$.

Since $S(\lambda)=S(\sigma) \cap \Lambda^{q}$, we see that $S(\sigma)$ has only a finite number of points in the hyperplane $n \cdot b=0$, and a suitable translation of $S(\sigma)$ by a vector in this hyperplane results in a singular measure $\sigma_{1} \in \mathscr{F}\left(T^{p}\right)$ which has $\hat{\sigma}_{1}(n)=0$ in a set $Q$ which satisfies the hypotheses of the Helson-Lowdenslager theorem, but which has $\hat{\sigma}_{1}(0)=1$.

This contradiction shows that $S(\mu)$ is finite, and the proof is complete. 


\section{Remarks.}

5.1 In the preceding section we have determined all idempotent measures on $T^{r}$, and incidentally also on all groups of the form $T^{r}+F$, where $F$ is any finite abelian group. We note that these groups are the only compact abelian groups which have no infinite totally disconnected subgroups.

Indeed, if $G$ is not $T^{r}+F$, then its dual group $\Gamma$ is not finitely generated (since every finitely generated abelian group is a direct sum of cyclic groups), and the well-known fact that a compact group is totally disconnected if and only if its dual group is a torsion group shows that the above-mentioned proposition is equivalent to the following purely algebraic theorem (compare 1.4 (a)).

5.2 THEOREM. If $G$ is an abelian group which is not finitely generated, then $G$ can be mapped homomorphically onto an infinite torsion group.

Proof. If $G$ has finite rank $p$, let $\left\{x_{1}, \cdots, x_{p}\right\}$ be an independent set in $G$. Factoring out the group generated by $x_{1}, \cdots, x_{p}$ gives a torsion quotient group, and the latter is infinite, since $G$ would otherwise be finitely generated.

If $G$ has infinite rank, let $\left\{x_{1}, x_{2}, x_{3}, \cdots\right\}$ be an independent set in $G$, and let $H$ be the group generated by $\left\{x_{n}\right\}$. Every $x \in H$ has a unique representation

$$
x=\sum_{1}^{\infty} a_{n}(x) x_{n},
$$

where the coefficients $a_{n}(x)$ are integers; for each $x$, only finitely many $a_{n}(x)$ are different from 0 .

Let $\left\{t_{n}\right\}$ be a sequence of distinct rational numbers, $0<t_{n}<1$, and define

$$
h(x)=\sum_{1}^{\infty} a_{n}(x) t_{n}
$$

It is clear that $h$ is a homomorphism of $H$ into the group $Y$ of the rationals modulo the integers, and since $h\left(x_{n}\right)=t_{n}, h(H)$ is infinite. Since $Y$ is divisible, $h$ can be extended to a homomorphism of $G$ into $Y$ [5; p. 11]. Since $Y$ is a torsion group, the homomorphism $h$ has the desired properties.

We conclude with a result which gives further support to the conjecture stated in 1.5.

5.3 THeOREM. Suppose $G$ is compact and connected, and $G$ is associated with an irreducible $\mu \in \mathscr{I}(G)$. Then $S(\mu)$ is a finite set. 
We shall omit the details of the proof, and merely give an outline: If $S(\mu)$ is infinite, then $S(\mu) \cap \Gamma_{0}$ is infinite, for some countable subgroup $\Gamma_{0}$ of $\Gamma$. Since $G$ is connected, $\Gamma$ has no element of finite order, and $\Gamma_{0}$ is isomorphic to a subgroup of the real line. If $A$ is any finitely generated subgroup of $\Gamma_{0}$, then $S(\mu) \cap \Lambda$ is finite, by the results of Section IV, and $I_{0}{ }_{0}$ is the union of a countable increasing sequence of such A's. A translation argument, combined with a generalized version of the Helson-Lowdenslager Theorem (Section 6 of [3]) now leads to a contradiction, as in Section IV.

PoSTSCRIPT (added in proof). About six months after the completion of this paper, P. J. Cohen has succeeded in proving the conjecture made in paragraph 1.5 in its full generality.

\section{REFERENCES}

1. Henry Helson, Note on harmonic functions, Proc. Amer. Math. Soc. 4 (1953), 686-691.

2. , On a theorem of Szegö, Proc. Amer Math. Soc. 6 (1955), 235-242.

3. ...., and David Lowdenslager, Prediction theory and Fourier series in several variables, Acta Math., 99 (1958), 165-202.

4. Edwin Hewitt, Remarks on the inversion of Fourier-Siieltjes transforms, Annals of Math. 57 (1953), 458-474.

5. Irving Kaplansky, Infinite Abelian groups, Ann Arbor, 1954.

6. Walter Rudin, The automorphisms and the endomorphisms of the group algebra of the unit circle, Acta Math. 95 (1956), 39-55.

UNIVERSITY OF ROCHESTER 



\section{PACIFIC JOURNAL OF MATHEMATICS}

\section{EDITORS}

\section{David Gilbarg}

Stanford University

Stanford, California

R. A. Beaumont

University of Washington

Seattle 5 , Washington
A. L. Whiteman

University of Southern California

Los Angeles 7, California

L. J. PAIGE

University of California

Los Angeles 24, California

\section{ASSOCIATE EDITORS}
E. F. BECKENBACH
C. E. BURGESS
E. HEWITT
A. HORN

\author{
V. GANAPATHY IYER \\ R. D. JAMES \\ M. S. KNEBELMAN \\ L. NACHBIN
}
I. NIVEN
E. G. STRAUS
T. G. OSTROM
H. L. ROYDEN
G. SZEKERES
M. M. SCHIFFER
F. WOLF
K. YOSIDA

\section{SUPPORTING INSTITUTIONS}

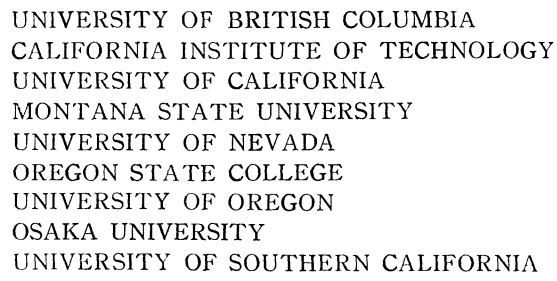

UNIVERSITY OF BRITISH COLUMBIA CALIFORNIA INSTITUTE OF TECHNOLOGY UNIVERSITY OF CALIFORNIA MONTANA STATE UNIVERSITY UNIVERSITY OF NEVADA OREGON STATE COLLEGE UNIVERSITY OF OREGON OSAKA UNIVERSITY UNIVERSITY OF SOUTHERN CALIFORNIA

STANFORD UNIVERSITY

UNIVERSITY OF TOKYO

UNIVERSITY OF UTAH

WASHINGTON STATE COLLEGE

UNIVERSITY OF WASHINGTON

AMERICAN MATHEMATICAL SOCIETY CALIFORNIA RESEARCH CORPORATION HUGHES AIRCRAFT COMPANY SPACE TECHNOLOGY LABORATORIES

Printed in Japan by Kokusai Bunken Insatsusha (International Academic Printing Co., Ltd.), Tokyo, Japan 


\section{Pacific Journal of Mathematics}

\section{Vol. 9, No. 1 \\ May, 1959}

Julius Rubin Blum and Murray Rosenblatt, On the structure of infinitely

divisible distributions . ............................. 1

Robert Geroge Buschman, Asymptotic expressions for

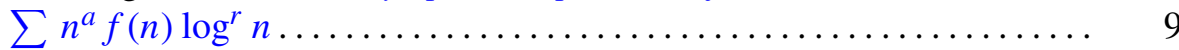

Eckford Cohen, A class of residue systems $(\bmod r)$ and related arithmetical

functions. I. A generalization of Möbius inversion .............. 13

Paul F. Conrad, Non-abelian ordered groups ................... 25

Richard Henry Crowell, On the van Kampen theorem............... 43

Irving Leonard Glicksberg, Convolution semigroups of measures ........ 51

Seymour Goldberg, Linear operators and their conjugates ............ 69

Olof Hanner, Mean play of sums of positional games .............. 81

Erhard Heinz, On one-to-one harmonic mappings ................ 101

John Rolfe Isbell, On finite-dimensional uniform spaces . . ........... 107

Erwin Kreyszig and John Todd, On the radius of univalence of the function

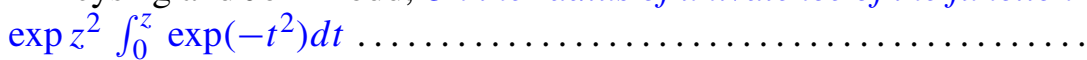

Roger Conant Lyndon, An interpolation theorem in the predicate

calculus......................................... 129

Roger Conant Lyndon, Properties preserved under homomorphism ........ 143

Roger Conant Lyndon, Properties preserved in subdirect products ....... 155

Robert Osserman, A lemma on analytic curves ................ 165

R. S. Phillips, On a theorem due to Sz.-Nagy..................... 169

Richard Scott Pierce, A generalization of atomic Boolean algebras ....... 175

J. B. Roberts, Analytic continuation of meromorphic functions in valued fields................................. 183

Walter Rudin, Idempotent measures on Abelian groups ................ 195

M. Schiffer, Fredholm eigen values of multiply-connected domains ........ 211

V. N. Singh, A note on the computation of Alder's polynomials ......... 271

Maurice Sion, On integration of 1-forms ...................... 277

Elbert A. Walker, Subdirect sums and infinite Abelian groups........... 287

John W. Woll, Homogeneous stochastic processes . .................. 293 